\title{
USING HAPTIC FEEDBACKS FOR OBSTACLE AVOIDANCE IN HELICOPTER FLIGHT
}

\section{Binet and T. Rakotomamonjy}

\author{
ONERA - The French Aerospace Lab \\ Systems Control and Flight Dynamics Department \\ 701 Base Aérienne, Salon de Provence 13300, France
}

\begin{abstract}
An obstacle avoidance function based on haptic feedback has been developed and tested on a simulation environment at ONERA. The objective was to calculate and provide efficient haptic feedback through active (motorized) sidesticks for the piloting task of a rotary wing (RW) aircraft, in the vicinity of visible and known obstacles, corresponding to emergency avoidance procedure, or navigation in a congested area. Two different methods have been designed to generate the force bias based on virtual force fields (VFF) surrounding obstacles and on a geometric approach (GA) combined with $\tau$-theory, respectively. Piloted simulations were performed in order to evaluate the benefits for obstacle avoidance.
\end{abstract}

\section{INTRODUCTION}

\subsection{Study Overview}

This paper presents the developments and the results obtained in the framework of two related research projects: AZUR (Autonomie en Zone Urbaine - Autonomy in Urban Zone) research project [1] led at ONERA — The French Aerospace Lab, and HOTAS (Haptic Obstacle and Terrain Avoidance System) developed in collaboration with DLR - German Aeronautics and Space Research Centre in Research Field 3 "Smart Rotorcraft" [2]. The objective of this study is to calculate and provide efficient haptic feedback through active (motorized) sidesticks for the piloting task of an RW aircraft, in the vicinity of visible and known obstacles of various types. The haptic feedback should provide the pilot with some assistance regarding the avoidance of those obstacles: although he is fully aware of their situation, some highly stressful and/or demanding piloting situations can lead to a wrong appreciation of the relative distances between the

(C) The Authors, published by EDP Sciences. This is an open access article distributed under the terms of the Creative Commons Attribution License 4.0 (http://creativecommons.org/licenses/by/4.0/). 
helicopter elements (in particular, for configurations where the pilot can hardly see the rotor blades) and the surrounding buildings, cliffs, walls, etc.

Developments and evaluations were done in simulation using a 10-ton class helicopter model (nevertheless, the force feedback functions developed could be transposed to unmanned aerial vehicle (UAV) obstacle navigation as well). The evaluations were done with an UAV obstacle field navigation benchmark proposed by U.S. Army [3] and used in the other activities of the AZUR project. The sizes and distances between obstacles were adapted to a helicopter with a 16-meter rotor diameter. The integration of rotorcraft flight model and obstacle map was done on PycsHel, the prototyping and real-time piloted simulation environment at ONERA Salon de Provence Research Facility.

In an initial approach, distance measurements (using telemetry sensors modelization) would be used in order to assess in real time the actual position of the obstacles in the simulation environment. However, the performance of the detection was closely dependent on the terrain database used (type of surfaces, etc.) As a consequence and in order to focus the evaluation on the haptic assistance by itself, it has been assumed for the piloted simulations that obstacles positions were precisely known in advance.

A few simple obstacle benchmarks were tested, corresponding to emergency avoidance procedure or navigation in a congested area. A specific scenario has been developed and tested, corresponding to a very simple urban environment. Two different logics have been used in order to generate force feedbacks on the cyclic active sidestick. These force feedback laws were implemented on PycsHel prototype and evaluated in piloted simulations on the different obstacle field benchmarks.

\subsection{Previous Works}

Previous studies led at ONERA on the development of pilot assistance through tactile feedbacks have considered two different scenarios: maintaining a flight at moderate to high speeds in a corridor (materialized with two parallel rows of trees (Fig. 1a) and flying over a last-minute obstacle.

In the first case, different tactile cues on the lateral control axis were implemented as a function of the proximity with the corridor walls: stick vibration, soft-stop, and force bias. These first tests showed that using stick vibration, the pilot was warned of the proximity of trees but vibrations were not directional. Increasing or decreasing the frequency and/or the amplitude with the proximity of the obstacle can help. But at a constant distance of the obstacle, the pilot had no information about the right way to move the stick. Using soft-stops prevented the pilot to continue to move the stick in the "wrong" direction, but force bias was the most comprehensive and intuitive feedback, generating a force pushing the stick in the opposite direction of the danger. 


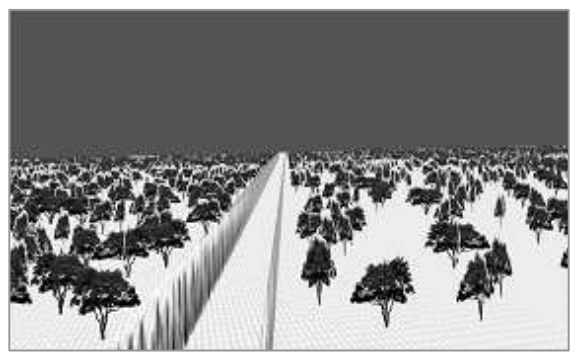

(a)

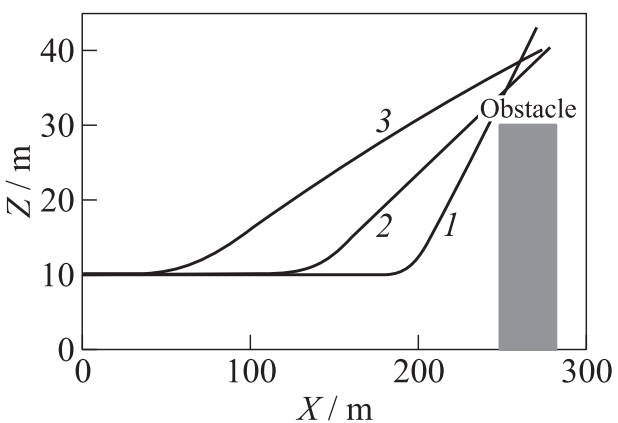

(b)

Figure 1 Overview of previous results: flying into corridor $(a)$ and vertical avoidance through collective stick $(b)(1-30 \mathrm{~km} / \mathrm{h} ; 2-60$; and $3-100 \mathrm{~km} / \mathrm{h})$

For the second scenario, a simple test case corresponding to a fly-over obstacle avoidance has been investigated through offline simulations. The result is a required command on the collective leading to an increase of the vertical speed. The required command has been inspired by previous works on $\tau$-theory and time to contact $[4,5]$. By applying the same principles and notations, it can be shown that the distance at which the collective has to be increased is given by

$$
D_{\text {react }}=\tau V_{H}
$$

where $\tau$ is the (estimated) time to contact; and $V_{H}$ is the horizontal speed of the helicopter. Then, the required climb slope is given by

$$
\gamma_{\mathrm{cons}}=\frac{H_{\mathrm{obs}}+\Delta H_{\mathrm{obs}}-H_{H}}{D_{\text {react }}}
$$

and, subsequently, the required collective level $\theta_{0}$ necessary to reach this slope is obtained through the following equations:

$$
\begin{aligned}
& \bar{\gamma}=-\left[1-\left(\frac{t}{\tau}\right)^{2}\right]^{1 / k} ; \\
& \bar{\gamma}^{\prime}=\left(\frac{2 t}{k \tau}\right)\left[1-\left(\frac{t}{\tau}\right)^{2}\right]^{1 / k-1} ;
\end{aligned}
$$




$$
\begin{aligned}
\bar{\theta}_{0} & =1-\left(\frac{1}{\tau Z_{w}}\right) \bar{\gamma}+\bar{\gamma}^{\prime} ; \\
\theta_{0 f} & =V_{H} \gamma_{\text {cons }} \frac{Z_{w}}{Z_{\theta 0}} \\
\theta_{0} & =\bar{\theta}_{0} \theta_{0 f}
\end{aligned}
$$

where $k$ is the coupling constant between the actual motion and a "guide motion" (see [5]); and $Z_{w}$ and $Z_{\theta 0}$ are, respectively, the heave damping derivative and heave control sensibility derivative, depending on the helicopter characteristics and current horizontal speed.

Figure $1 b$ shows three different trajectories followed by the helicopter model for three different speeds. The beginning of the avoidance maneuver depends on the horizontal speed. Here, the generated command is directly used as the collective command, it is not a force/displacement law which could be used by the active stick. Nevertheless, this would be the base of such law. The required collective level given by the algorithm would be used in the generation of a force bias or a soft-stop function.

Depending on the character of emergency, it could be necessary to take into account the helicopter limits in terms of overtorque (due to the increase of the collective) or to verify the feasibility of the maneuver (helicopter maximum rate of climb). Moreover, an action on the longitudinal cyclic would also be useful in such maneuver and a specific haptic feedback on this axis should be developed.

\section{OVERVIEW OF THE SIMULATION ENVIRONMENT}

\subsection{PycsHel Prototyping and Simulation Environment}

The developments and simulations were led through the PycsHel prototyping environment, part of LabSim simulation facilities at ONERA center of Salon de Provence. This consists in:

- 2 seats replicating the standard inner disposition of a helicopter cockpit;

- a 270 degree field-of-view display, obtained with 3 white walls and 3 videoprojectors mounted on the ceiling;

- standard (passive) flight controls for the cyclic, collective, and pedal inputs on the left seat; 
- motorized programmable active sidesticks on the right seat (cyclic and collective controls), manufactured by WITTENSTEIN, allowing to give a haptic feedback to the pilot;

- custom-made visual engine, based on open-source three-dimensional (3D) graphics toolkit OpenSceneGraph;

- the capacity to run in real-time high fidelity helicopter flight dynamics code (HOST [6] and others); and

- entirely open and programmable environment, for the inclusion of specific models developed using Matlab/Simulink, C++, FORTRAN, etc.

\subsection{Description of the Map and Obstacles}

A specific map has been designed by placing various 3D obstacles on a plain, flat ground. Those obstacles are derived from a benchmark proposed by the U.S. Army to evaluate the performance of guidance and navigation algorithms of

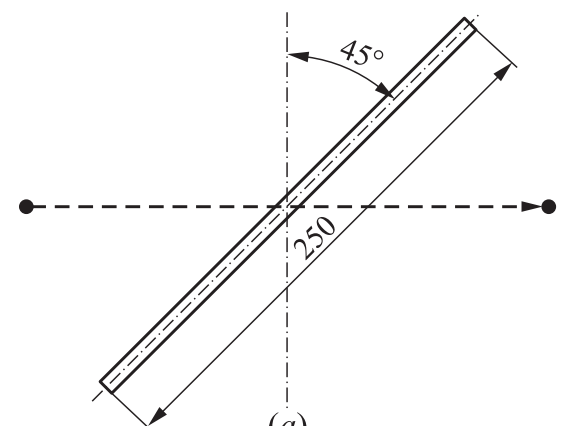

(a)

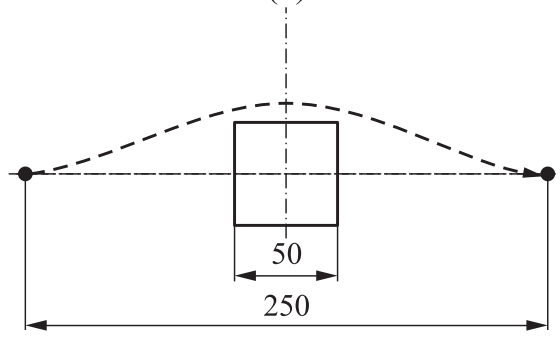

(b)

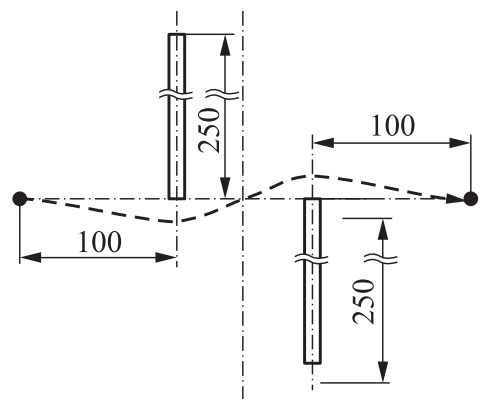

(c)

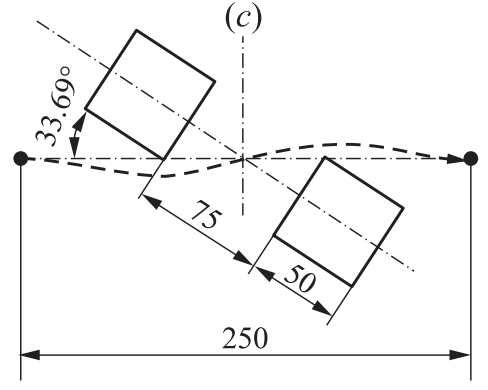

(d)

Figure 2 Topology of the obstacles used in the simulation environment (top view): (a) wall obstacles; (b) cube obstacles; $(c)$ wall baffle obstacles; and $(d)$ cube baffle obstacles. Dimensions are in meters 
UAVs in the vicinity of obstacles [3]. Since the dimensions and maneuverability of the helicopter considered here are rather different from those of the UAVs, the benchmark was devised for, the obstacles have been resized at scale (Fig. 2). Wall obstacle has been implemented in the terrain database but no haptic function has been developed yet for this specific task.

The dashed lines represent the prescribed flight paths for the obstacle avoidance. For the case of the cube, wall baffles and cube baffle obstacles, both flight paths and obstacle avoidance trajectories are considered in the horizontal plane only: the obstacles are supposed to be of infinite height and, thus, the avoidance cannot be done by flying above them.

The obstacles were recreated with a 3D modeling software, accompanied by a "landing strip" at the root which indicates the orientation of the prescribed ap-

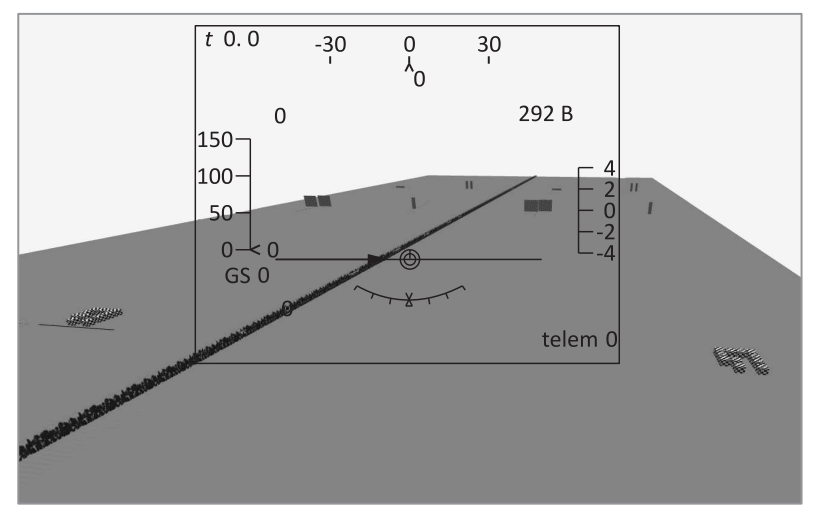

Figure 3 Global perspective view of the whole map

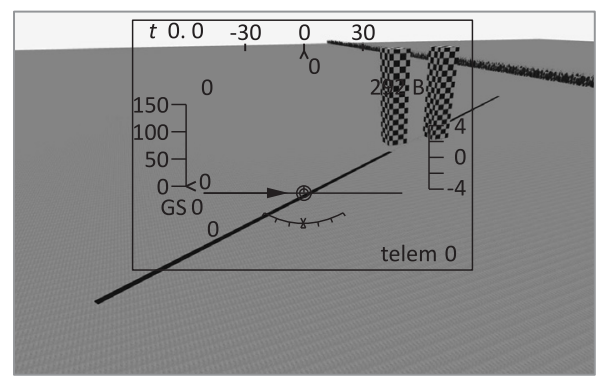

(a)

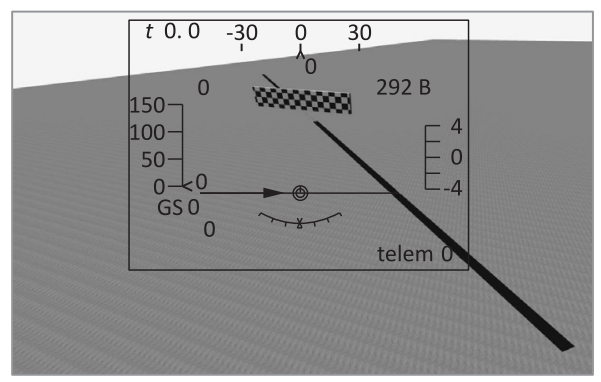

(b)

Figure 4 Simulation in-flight view of different obstacles: $(a)$ cube baffle obstacle; and $(b)$ single wall obstacle 


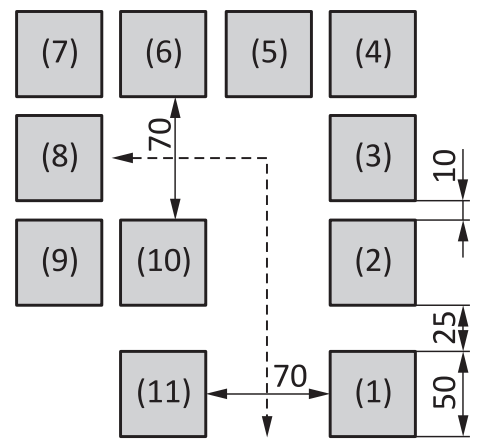

(a)

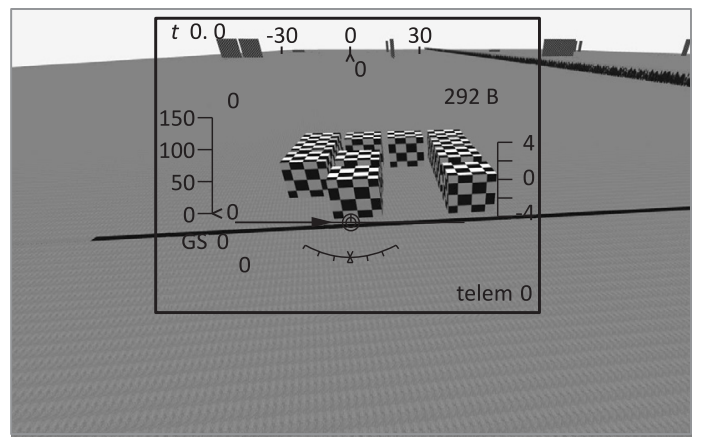

(b)

Figure 5 Definition of the confined zone: (a) top view; and (b) in-flight front view. Dimensions are in meters

proach. For the convenience of the pilot, the cube-shaped obstacles were dilated along the vertical axis so that it is not necessary to maintain a perfectly constant flight altitude during the approach to avoid hitting the ground. However, the denomination "cube" and "cube baffle" (originally related to the ground print of the obstacles) will still be used in this paper, even if they appear as "towers," or rectangular parallelepipeds (Figs. 3 and 4).

In addition to the sets of obstacles inspired from the benchmark in Fig. 2, another specific set has been designed in order to simulate a confined zone, such one a pilot can encounter when flying in densely urbanized areas or mountain regions. This set, visible in Fig. 5, is comprised of 11 identical cubic blocks (edge length: $50 \mathrm{~m}$, same ground print as the single "cube" obstacle) arranged in a cul-de-sac, with 2 blocks defining a small corridor at the entrance. This set will be used for descent and landing tasks, where the pilot will have to approach at constant slope, decelerate, turn, and maintain a stabilized hovering spot in front of the bottom of the zone (the interaction between landing gears and the ground has not been reimplemented yet into the simulator; this is why, a complete landing until touchdown will only be possible in future versions of the simulation environment).

\section{HAPTIC FORCE FOR OBSTACLE AVOIDANCE}

\subsection{Active Inceptors and Force Feedback}

The active sidestick/inceptor is a control input device that generates the mechanical forces perceived by the pilot using electric motors. This allows a high 


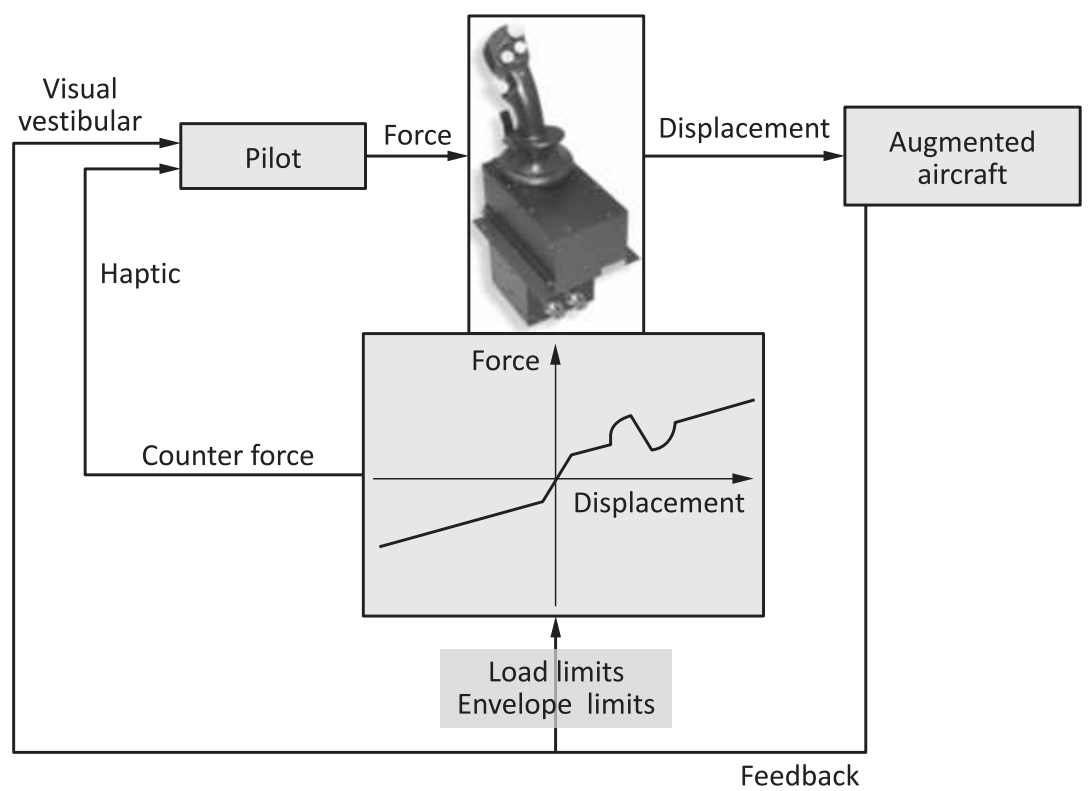

Figure 6 The active inceptor in the pilot control loop

degree of freedom in the design of the Human/Machine interface. Not only the traditional spring-mass-damper forces are emulated, but also a wide range of additional tactile (or haptic) cues is possible.

Figure 6 shows the general signal and information flow when a compliant active inceptor is added to the system \{Pilot $\Leftrightarrow$ Augmented aircraft $\}$ [7]. The pilot generates a force and the internal control scheme following the inceptor force-displacement algorithm moves the stick to the position where the force is prescribed [8]. The transitional behavior of the movement is normally a secondorder system (mass, spring, and damper). On top of this, functions like detents, breakout, softstops, etc. can be placed to indicate specific events to the pilot. It means, in addition to the classical visual and vestibular feedback to the pilot, that a haptic feedback is added.

For obstacle avoidance, the cueing function works by giving the pilot a force bias. This bias was computed (as described in the next chapters) and added to the main force/displacement curve settled on the cyclic stick. As it can be seen in Fig. 7, depending on the sign of the bias, the main curve is shifted up or down the force axis. If the operator does not counteract $(F=0)$, the stick will move on the right (for a negative bias value) or on the left (for a positive bias value). In order to maintain the position of the stick, the operator will have to increase the force he/she is applying on the stick. 


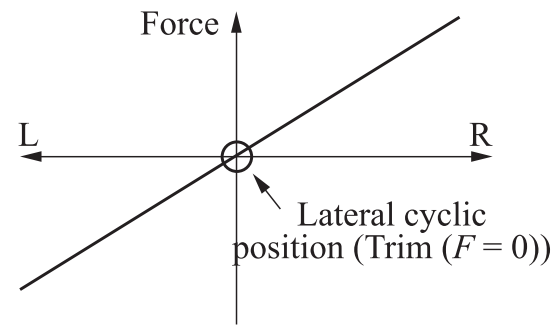

(a)

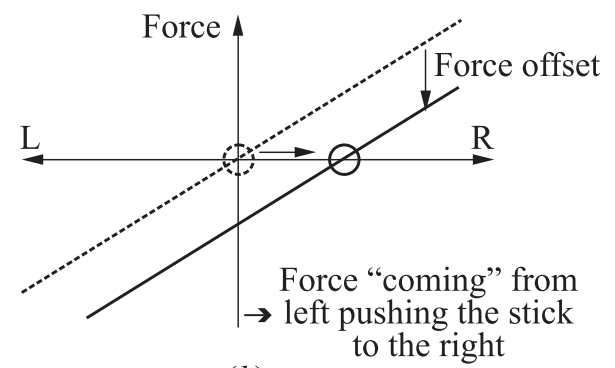

(b)

Figure 7 Force bias feedback on active stick: $(a)$ no force bias; and (b) force bias to the right

The next subsections detail the two different techniques developed in order to calculate the force feedback transmitted to the pilot through the active sidestick. First, a force-field method where the gradients of a potential proximity function are calculated; then, a geometric method based once again on $\tau$-theory $[4,5]$.

For both methods, three different frames will be used, in which the coordinates of the rotorcraft and the obstacles will be expressed. All frames are supposed orthonormal, direct-oriented:

$-\mathcal{R}_{0}=\left(O_{0}, \vec{x}_{0}, \vec{y}_{0}, \vec{z}_{0}\right):$ reference, inertial frame, attached to the ground. Here, $O_{0}$ is the center of the map; $\vec{x}_{0}$ points towards north; $\vec{y}_{0}$ points towards east; and $\vec{z}_{0}=\vec{x}_{0} \wedge \vec{y}_{0}$ points towards the center of the Earth;

- $\mathcal{R}_{a}=\left(O_{a}, \vec{x}_{a}, \vec{y}_{a}, \vec{z}_{a}\right)$ : aerodynamical frame, defined by the helicopter air speed $\vec{V}$. Here, $O_{a}$ is the center of the airframe; $\vec{x}_{a}$ points along $\vec{V}\left(\vec{x}_{a}\right.$ $=\vec{V} /\|\vec{V}\|) ; \vec{y}_{a}$ is orthogonal to $\vec{x}_{a}$ and points towards the right (from a pilot point of view); and $\vec{z}_{a}$ points downwards (note: frame is undefined if $\vec{V}=\overrightarrow{0}$ ); and

- $\mathcal{R}_{b}=\left(O_{b}, \vec{x}_{b}, \vec{y}_{b}, \vec{z}_{b}\right)$ : aircraft body frame, attached to the airframe. Here, $O_{b}$ is the center of the airframe; $\vec{x}_{b}$ points from aft to nose; $\vec{y}_{b}$ is orthogonal to $\vec{x}_{b}$ and points towards the right (from a pilot point of view); and $\vec{z}_{b}$ points downwards.

\section{$3.2 \quad$ Force-Field Approach}

The first method proposed for the computation of haptic feedback is based upon artificial or virtual force fields. The concept of VFFs has been widely used in robotics, in particular, for the guidance and path planning of autonomous 
robots [9-11] and was also recently improved through hybridation with other techniques $[12,13]$.

The objective is to translate the information of proximity of an obstacle into another dimension - in this case, a force vector — so that the minimum energy configuration of the system will be equivalent to the "safest" trajectory.

Let $n$ be the total number of single buildings (for example, a cube baffle consists in two single cubes). The position and size of each building $i \in \llbracket 1 ; n \rrbracket$ are supposed entirely known. In this case, the simplest approach consists in converting the proximity towards a given building as a potential-like function $z(x, y)$ which decreases uniformly as the mobile gets farther from it.

In the first approach, the height of the buildings is not considered, since an avoidance by flying above the obstacle will not be studied here. The ground trace of a building will be approximated by an ellipsis - even if they have a rectangular section - whose half axis parameters $a_{i}$ and $b_{i}$ define the aspect ratio (square for a cube, elongated for a wall).

With the extra hypothesis that the axes of the ellipsis have to remain parallel to $\vec{x}_{0}$ and $\vec{y}_{0}$ axes, the following candidate potential function satisfies all above requirements:

$$
\begin{aligned}
\phi_{i}(x, y) & =\frac{k_{i}}{\sqrt{\left(x-x_{i}\right)^{2} / a_{i}^{2}+\left(y-y_{i}\right)^{2} / b_{i}^{2}+k_{i}^{2}}} ; \\
\phi(x, y) & =\max _{i \in \llbracket 1 ; n \rrbracket} \phi_{i}(x, y) .
\end{aligned}
$$

This potential is normalized and is equal to 1 at the center $\left(x_{i} ; y_{i}\right)$ of the building. Parameter $k_{i}$ defines the width of the potential surface and thus corresponds to the ground area of the building.

The array showing the optimal direction to get away from the obstacle is given in ground frame $\mathcal{R}_{0}$ by the gradient of $\phi$ :

$$
\left(\begin{array}{l}
p_{x} \\
p_{y}
\end{array}\right) \triangleq-\nabla \phi=\left(\begin{array}{r}
-\frac{\partial \phi}{\partial x} \\
-\frac{\partial \phi}{\partial y}
\end{array}\right) .
$$

Intuitively, this information should be given to the pilot through the cyclic longitudinal and lateral axes, in order to help him/her to avoid the closest obstacle. Thus, one can define in the first approach the Force Offset input for the active stick as being directly proportional to the components of $-\nabla \phi$ expressed in the helicopter body frame $\mathcal{R}_{b}$. Let $M(\psi)$ be the matrix corresponding to the rotation from frame $\mathcal{R}_{0}$ to $\mathcal{R}_{b}$ along vertical axis: 


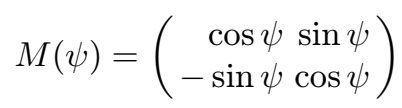

where $\psi$ is the heading angle $\left(\psi=0\right.$ when $\left.\vec{x}_{b}=\vec{x}_{0}\right)$.

Then, the forces to be exerted by the active sidestick can be given as

$$
\left(\begin{array}{c}
F_{x} \\
F_{y}
\end{array}\right)=\lambda M(\psi)\left(\begin{array}{c}
p_{x} \\
p_{y}
\end{array}\right)
$$

where

$$
\lambda=\left(\begin{array}{cc}
\lambda_{x} & 0 \\
0 & \lambda_{y}
\end{array}\right)
$$

is the coefficient matrix for adjusting the amplitude of the forces along each axes.

In addition to this, it would appear more natural for the pilot if the haptic feedback decreases whenever the helicopter faces away from the obstacle (independently to the position within the force field). To do so, a weighting function is added to $F_{x}$ and $F_{y}$.

Let $\beta=\angle\left(-\nabla \phi, \vec{V}_{H}\right)$ be the angle between the proximity gradient and the ground speed. It can be shown that

$$
\beta=\pi-\operatorname{atan}^{2}\left(\frac{\partial \phi}{\partial y}, \frac{\partial \phi}{\partial x}\right)+\chi_{H}
$$

where $\chi_{H}$ is the route angle in the horizontal place between $\vec{x}_{0}$ and the ground speed $\vec{V}_{H}$. Then, the following candidate weighting function can be used: $\sin ^{4}(\beta / 2)$, which equals 0 for $\beta=0$ (in this case, the helicopter is getting away from the obstacle; so, no force feedback is applied) and 1 for $\beta= \pm \pi$ (here,

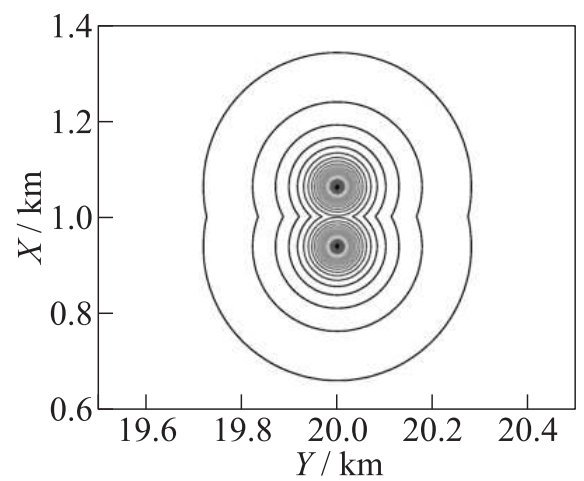

(a)

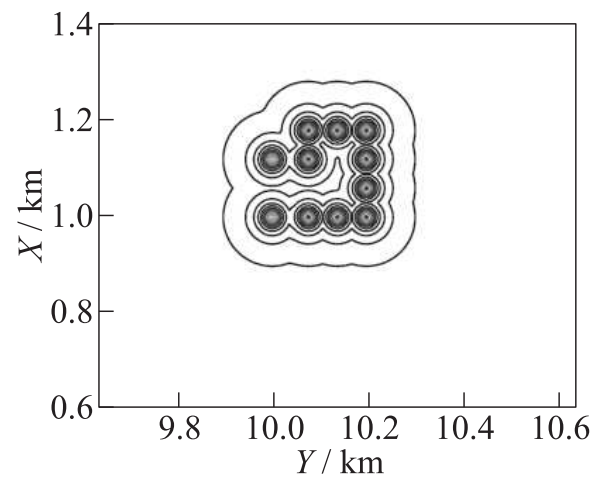

(b)

Figure 8 Examples of generated VFFs for two different obstacle sets: (a) cube baffle; and $(b)$ confined zone 
the force feedback should have its maximal value). Finally, the complete force feedback law can be expressed as

$$
\left(\begin{array}{c}
F_{x} \\
F_{y}
\end{array}\right)=\lambda \sin ^{4}\left(\frac{\beta}{2}\right) M(\psi)\left(\begin{array}{c}
p_{x} \\
p_{y}
\end{array}\right) .
$$

The numerical implementation of this force field approach for offline and online (within the PycsHel environment) simulations requires an exact knowledge of all obstacles position and size as it has been mentioned earlier. For this reason, the complete gradient field map $\nabla \phi(x, y)$ could also be computed offline and used in the simulations as tabulated values (Fig. 8).

\subsection{Geometric Approach}

A second logic has been investigated to generate force feedbacks, hereafter called geometric approach. It is composed of two different algorithms, the first one is dedicated to the collision evaluation and the second to the force feedback generation.

First, a simple algorithm providing the detection of a collision risk has been designed. It is based on a circle (defined by a radius $R_{H}$ ) surrounding the helicopter and providing a safety margin around it independently of its attitude. A similar circle (of radius $R_{O}$ ) is defined around the obstacle(s). There is a risk of conflict if the helicopter speed vector is included in the angular sector $2 \delta$ determined by the tangents to circles as shown in Fig. $9 a$.

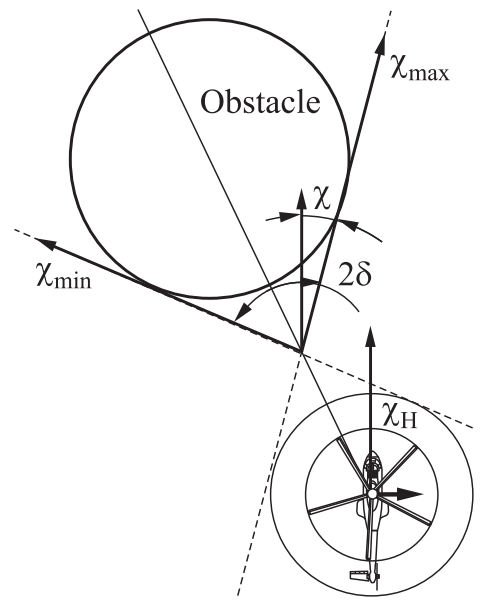

(a)

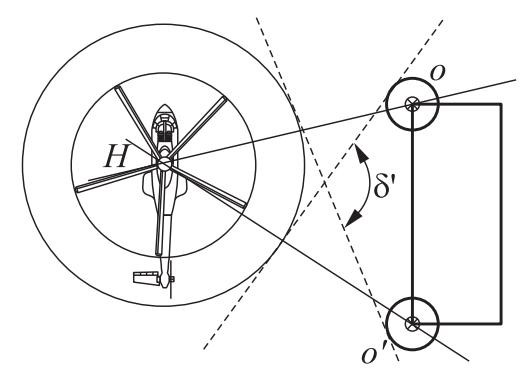

(b)

Figure 9 Collision estimation: ( $a$ ) front obstacle; and (b) large side obstacle 


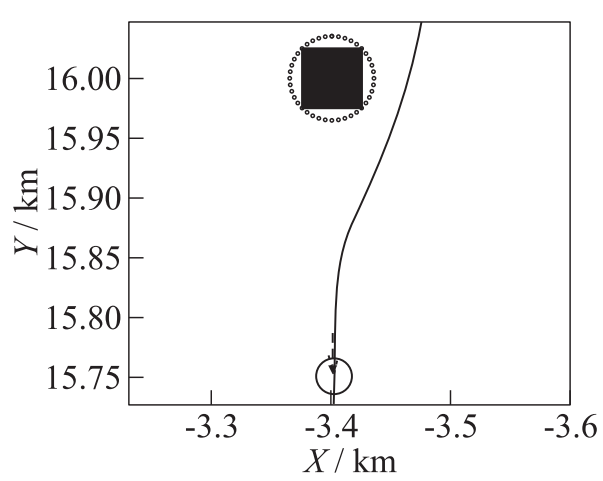

(a)

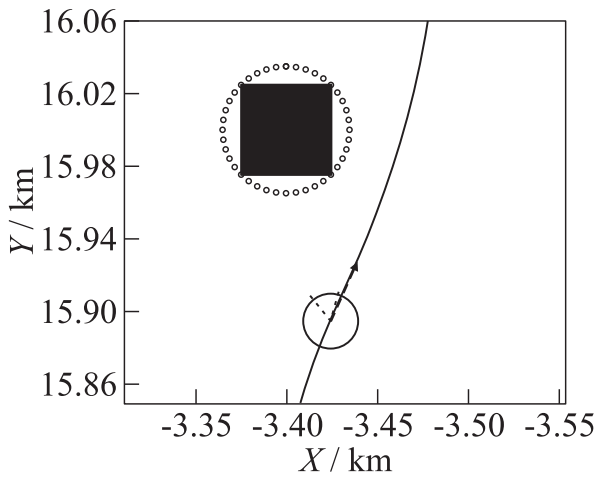

(b)

Figure 10 Collision risk in front of the cube benchmark: (a)collision risk if the trajectory remains unchanged and $(b)$ collision avoided

The angular sector is given by the following equations:

$$
\begin{aligned}
K & =\frac{1}{1+R_{H} / R_{O}} ; \quad \overline{H I}=K \times \overline{H O} \\
\overline{T I} & =\sqrt{\overline{H I}^{2}-{\overline{R_{H}}}^{2}} ; \quad \delta=\arctan \left(\frac{R_{H}}{\overline{T I}}\right) \Rightarrow\left[\chi_{\min } ; \chi_{\max }\right] .
\end{aligned}
$$

The deviation angle $\chi$ is obtained by comparisons between the helicopter route angle $\chi_{H}$ and $\chi_{\max }$ and $\chi_{\min }$. As explained later, the force feedback is computed in order to change the helicopter trajectory in the direction where the route deviation is minimum.

In Fig. $10 a$, the helicopter speed vector (dashed arrow) is included in the angular sector formed by $\chi_{\min }$ and $\chi_{\max }$ (dotted arrows); so, there is a risk of collision. In Fig. 10b, one can see that the dashed arrow is outside the angular sector, there is no more risk in this situation.

This algorithm can also be used for large obstacles, by defining circles at different locations on the obstacles (as shown in Fig. $9 b$ ). It can also be adapted to close isolated obstacles: in such a case, two angular sectors are determined simultaneously corresponding to the two different obstacles. Weighting is then applied when generating the force feedback to take into account the proximity of different obstacles.

Once the risk of collision has been estimated, a force feedback is applied on the lateral cyclic control input. For that purpose, the distance between the circles related to the helicopter and the obstacle is calculated. The distance at which the pilot should feel the information on the stick is given by $D_{\text {react }}$ : 


$$
\begin{aligned}
D_{\text {sphere }} & =H O-R_{H}-R_{O} ; \\
D_{\text {react }} & =\tau V_{H} .
\end{aligned}
$$

This is a way to generate haptic feedbacks at the time that the pilot would have start to change its trajectory by himself. This approach is inspired again from the $\tau$-theory. Depending on the helicopter/obstacle distance, the first coefficient $K_{f}$ is computed as follows:

$$
K_{f}= \begin{cases}30, & \text { if } D_{\text {sphere }}<0 \\ 0, & \text { if }\left|D_{\text {sphere }}\right|>D_{\text {react }} \\ 30-30 \sin \left(\frac{\pi}{2} \frac{D_{\text {sphere }}}{D_{\text {react }}}\right)^{2} & \text { else. }\end{cases}
$$

Finally, the force bias used as feedback is given by the following formula, depending of the deviation angle $\chi$ between the helicopter route angle and the angular sector limits:

$$
F_{y}= \pm K_{f}\left(\frac{\chi}{10}\right)
$$

where the sign depends on a right or left deviation.

\section{PILOTED EVALUATION RESULTS}

\subsection{Preliminary Remarks}

The experiments consisted in several runs of piloted simulation, starting from one point before the obstacle, with a specified position and velocity, and flying towards the obstacle with the general objective of performing an avoidance maneuver as "natural" as possible. Some specific trials were performed such as passing as close to the cube as possible or without counteracting the stick. Due to schedule constraints, professional helicopter pilots could not be available of this series of experiments. For this reason, all trials were performed by one nonprofessional pilot (ONERA flight dynamics engineer) who although has been used to flying helicopters in simulation.

For each obstacle set, several runs were performed with VFF, with GA, and also without any haptic feedback on the sidestick, for various initial velocities. For each run, all measured (forces and displacements of the sidestick) and simulated (aircraft position, velocity, etc.) variables were recorded continuously, at a sampling time $\Delta t=10 \mathrm{~ms}$ and were put together available as .csv files for later processing.

As the rotor disk is not visible in the visual environment and due to the lack of visual markers in the terrain database, it was very difficult to estimate 
the distance of the helicopter from the obstacle(s). This remark is true for all test cases performed in this study and, more generally, for all tests performed in simulators. Although the helicopter flight control system is equipped with high level piloting laws (ACAH - Attitude Command, Attitude Hold, RCAH Rate Command, Attitude Hold), all tests were performed by only using the SAS (Stability Augmentation System). For professional helicopter pilots, this would have no influence, but piloting and maintaining low speeds during the runs were more difficult in the considered case.

The influence of a degraded visibility environment (DVE) has also been investigated in some test cases, since the visual engine of the PycsHel simulator is also able to generate fog whose thickness and density parameters can be freely tuned.

\subsection{Cube Baffle}

The first task evaluated has been the cube baffle. The objective of the task was to follow the ground print and to pass between the obstacles. This task has been done for several initial values of helicopter speed which was supposed to be maintained constant during the run.

Figure 11 shows two helicopter trajectories without haptic feedback (Fig. 11a), and one trajectory with the GA (Fig. 11b). The initial speed here was $3-5 \mathrm{kts}$. The grey circles represent the helicopter rotor at the minimum distance with respect to each obstacle. The grey arrows represent the helicopter tail boom in this situation.

As explained before, it was very difficult to estimate the distance between the helicopter and the obstacle. Moreover, maintaining a very low speed required an

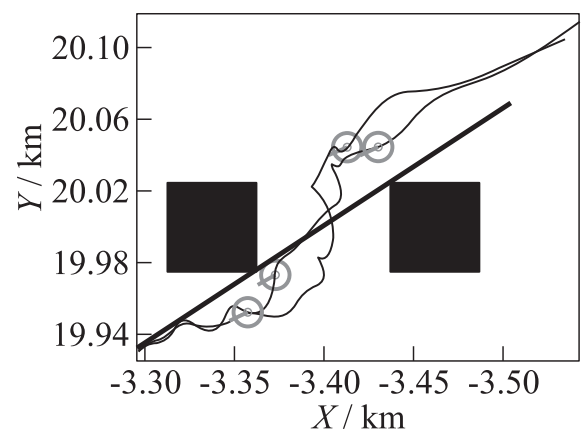

(a)

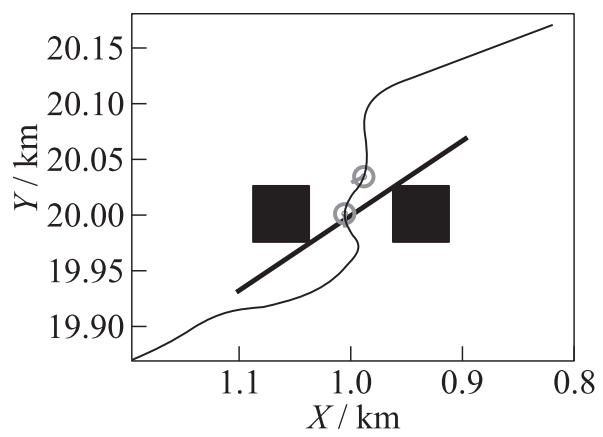

(b)

Figure 11 Cube baffle tests at 3-5 kts without force feedback (a) and with VFF feedback $(b)$ 


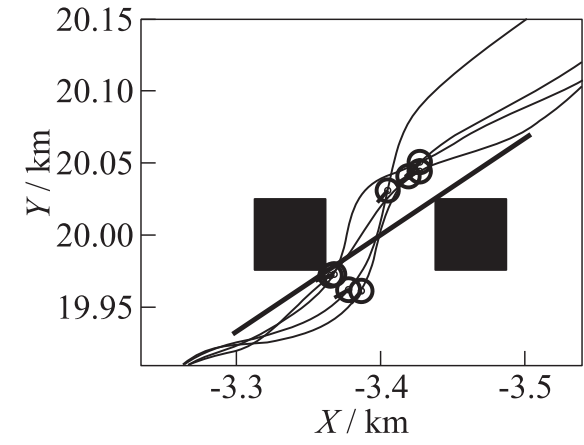

(a)

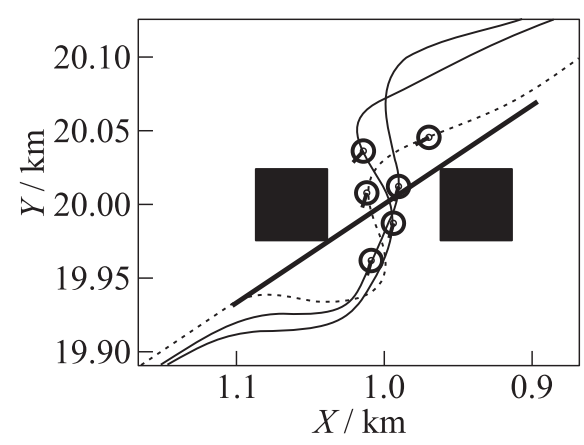

(b)

Figure 12 Cube baffle tests at 20 kts without force feedback $(a)$ and with VFF feedback (solid curves) and GA feedback (dotted curve) (b)

increased workload. Without haptic feedback, the minimum distance measured was $9.94 \mathrm{~m}$ compared to $27.18 \mathrm{~m}$ with haptic feedbacks. Feedbacks are felt early, leading the pilot to change the trajectory sooner than without feedback and increasing the safety margin around the first obstacle.

Figure 12 shows helicopter trajectories without haptic feedback (Fig. 12a) and with haptic feedbacks (Fig. 12b) at the initial speed of $20 \mathrm{kts}$. Once again, with no feedback, the helicopter collided the first obstacles two times. Using VFF, the force feedback was a bit too high and the pilot must counteract the stick movement. Passing near the second obstacle, the helicopter is "pushed away" with big changes in helicopter attitudes. But it allows a quick maneuver between the obstacles. Taking into account the previous runs, some modifications in the force bias computation were brought in the GA algorithm. Moreover, $\tau$ was reduced from 10 to $5 \mathrm{~s}$ and $K_{f}$ - from 30 to 10 . These modifications gave good results but some improvements could still be done (taking into account that the risk of collision with one of the obstacle is decreasing, for example).

These first evaluations led to the following preliminary conclusions:

- without haptic function, this task is not easy, especially for low speeds. The helicopter hit the first obstacle several times during the tests. It has to be mentioned that in a real flight test, the pilot would certainly take a wider safety margin and would avoid the first obstacle by changing his/her trajectory sooner;

- considering VFF approach, force feedbacks on the longitudinal axis are not always adapted, especially for speeds higher than $5 \mathrm{kts}$. They change the pitch attitude and stop the helicopter if the pilot does not react. Weighting 
coefficients $\lambda_{x}$ and $\lambda_{y}$ between lateral and longitudinal force feedback had to be slightly modified during the tests in order to increase the lateral force (leading to a lateral deviation). Finally, if the helicopter flies straight ahead of the obstacle, there is only a longitudinal force feedback which is not always adapted;

- the GA is not adapted to close obstacles. The risk of collision algorithm has been validated for two different obstacles, but the generation of the force bias was not adapted, leading to inconsistent forces on the stick. The time to contact parameter $(\tau)$ used in the force generation has to be reduced; and

- for both VFF and GA, haptic feedbacks give better results at higher speeds. Indeed, at very low speeds, force feedbacks have to be counteracted to smoothen changes in helicopter attitudes and speed variations causing an increase of the workload. This sometimes led to the feeling that the helicopter might be bumping from one obstacle to another.

\subsection{Cube}

Cube task has been the second test case. As it can be seen in Fig. $2 b$, the obstacle is relatively large and it can be seen from far away. For that specific task, finding a realistic scenario where the pilot is not aware of the obstacle is not easy. As it is impossible to estimate where the edge of rotor is located, the first piloting task chosen was to fly directly towards the obstacle and then to avoid it with a minimum passing distance. The initial helicopter speed was $75 \mathrm{kts}$ and the pilot was instructed to maintain this speed during the run.

Figure 13 shows helicopter trajectories without any feedback, with GA feedback, and with VFF feedback, respectively.

The minimum passing distance obtained in all runs for the different feedbacks was 12.47 (see Fig. 13a), 19.85 (see Fig. 13b), and $13.16 \mathrm{~m}$ (see Fig 13c). As the obstacle was seen from far away, the avoidance maneuver was generally started before feeling any feedback. Due to their formulations, feedbacks were felt earlier on the trajectory when using the GA, closer to the obstacle when using the VFF approach. Moreover, the dispersion of the minimum passing distances is reduced when using haptic feedbacks.

As it appeared that avoiding the cube was too easy, the second piloting task has been tested. Initial conditions remained the same but fog was introduced in the visual environment as shown in Fig. 14a. In these conditions, the obstacle could be seen at around $170 \mathrm{~m}$. As the helicopter speed was $75 \mathrm{kts}$, the time to impact was around $4.4 \mathrm{~s}$.

Figure $14 b$ shows helicopter trajectories without feedback (dashed curve), with VFF (solid curves), and with GA (dotted curves). Without force feedback, 


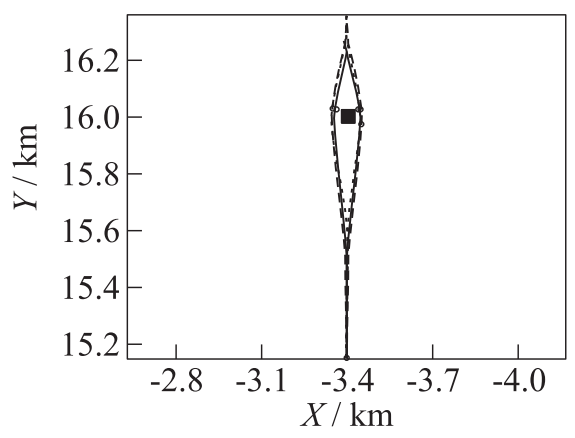

(a)

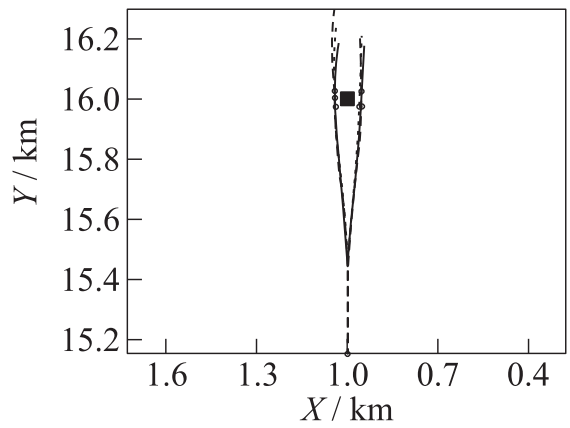

(b)

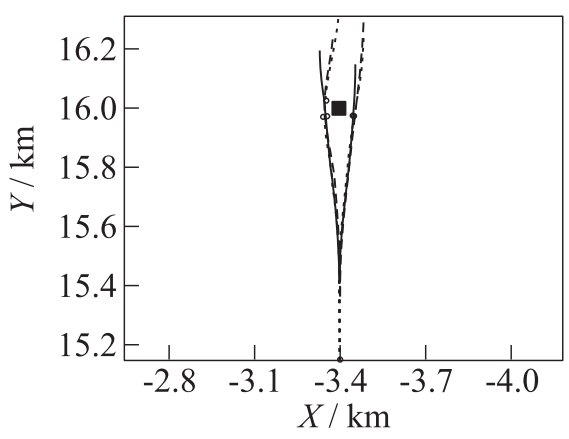

(c)

Figure 13 Cube avoidance tests without feedback $(a)$, with GA feedback $(b)$, and with VFF feedback $(c)$

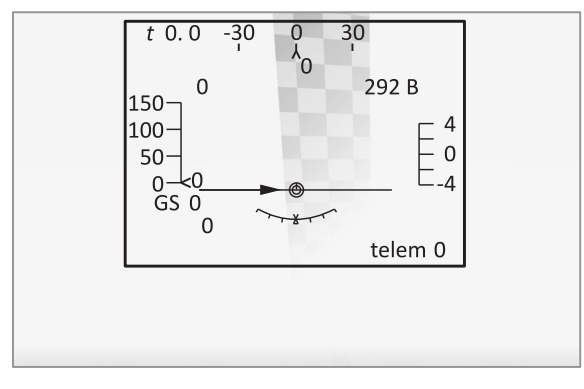

(a)

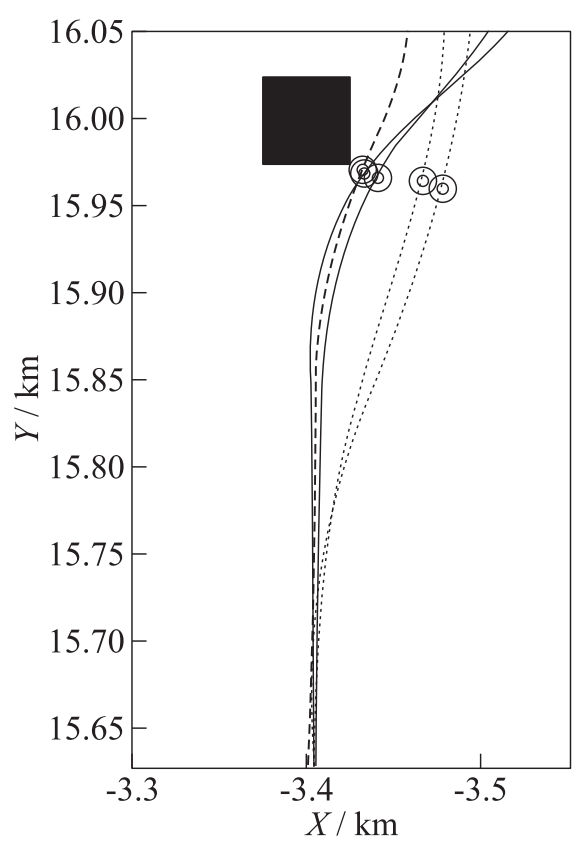

(b)

Figure 14 Cube obstacle in DVE (fog): (a) in-flight view; and (b) trajectories without feedback (dashed curve), with VFF feedback (solid curves), and with GA feedback (dotted curves) 
it is impossible to estimate the proximity of the obstacle and to begin the avoidance maneuver before seeing the cube. In that case, it is very difficult to avoid the obstacle at this speed.

For the computation of the force feedback following any of the two methods (VFF and GA), it has been supposed that the location of the obstacle was precisely known in advance, since it is not the object of the present study to model and simulate any position sensor or real-time mapping functionality.

Using haptic feedbacks, it is possible to inform the pilot before the obstacle can be seen. With GA, the force feedback is sent to the lateral cyclic as soon as the time to contact is $10 \mathrm{~s}$. It can be seen that it is sufficient to begin the avoidance maneuver in very safe conditions. For VFF, as force field is closer to the obstacle, the feedback is felt much more later. It is sufficient to avoid the obstacle but the passing distance is very low.

With no possibility to see the obstacle and this relative high speed, the force field should be adapted (enlarged) to this relative high speed in order to be felt sooner.

\subsection{Wall Baffle}

The third test case was dedicated to the wall baffle benchmark. As for the cube baffle tests, the objective was to follow the ground print and to pass between the obstacles at a constant speed. This task has been done for three different initial helicopter speeds $(10,20$, and $40 \mathrm{kts})$, supposed to be maintained during the run. This benchmark has not been evaluated with GA.

Figure 15 shows helicopter trajectories without feedback (left column) and with VFF (right column) at the initial speed of $10 \mathrm{kts}$.

At $10 \mathrm{kts}$ without feedback, there was no difficulty to perform the task (Fig. 15a). But once the first obstacle was passed, it was still difficult to estimate its proximity. Using VFF, the trajectory was changed by a slight right turn or sideslip in order to pass the first obstacle. Approaching the obstacles, the force feedbacks were mainly due to the second obstacle (on the longitudinal cyclic), with very few feedback on the lateral axis. The pilot had to counterbalance the longitudinal stick force in order to hold the speed. When passing between the obstacles, force bias was well sized.

At $40 \mathrm{kts}$ (Fig. 15b), it becomes necessary for the pilot to anticipate the change of trajectory early enough and to have a larger distance with respect to the first obstacle compared to the previous case. With VFF, when waiting for the feedbacks which are felt relatively close to the obstacles, the change in the trajectory is done later, which can, in turn, explain why the minimum distance from the second obstacle is shorter than without feedback. 

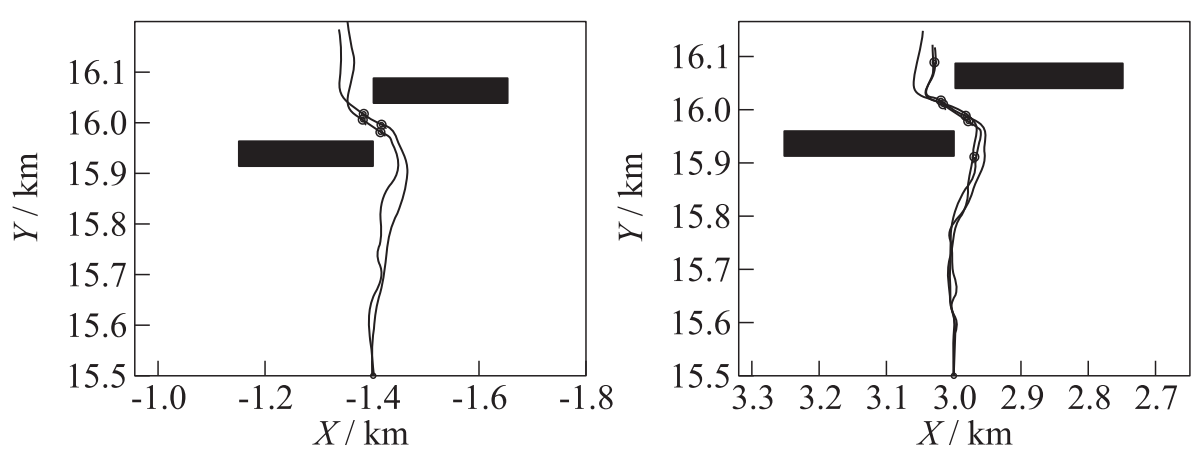

(a)

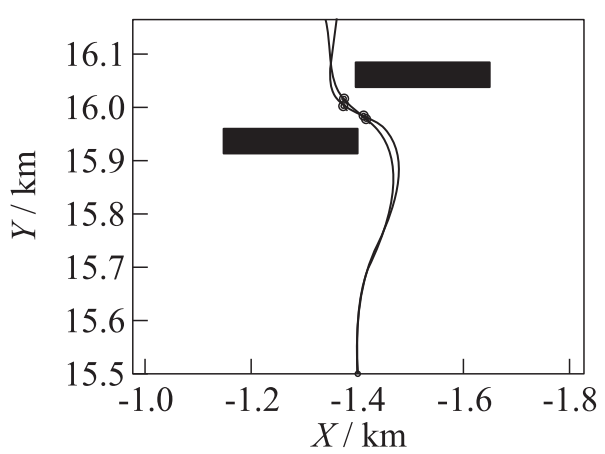

(c)

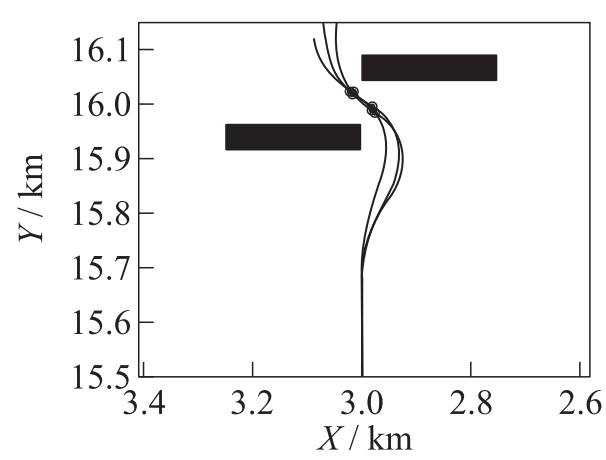

(b)

Figure 15 Wall baffle tests at $10(a)$ and $40 \mathrm{kts}(b)$ without force feedback (left column) and with VFF feedback (right column)

\subsection{Confined Zone}

The last piloted simulations were dedicated to the confined zone (see Fig. 5). The first test cases considered an initial approach on a constant glide of $-8^{\circ}$ at a constant horizontal speed of $40 \mathrm{kts}$. The goal was to enter the confined area and to make a left turn and to hover near the ground on the left part of the zone as close as possible to the wall. After that, to make a 180 degree turn, a right turn, and go out. The GA was not evaluated during these tests.

Figure 16 represents the helicopter trajectories when flying without haptic feedback (Fig. 16a) and with VFF feedback (Fig. 16b) for several runs (one color for each separate run).

When only considering the minimum distances from obstacles, the benefit of haptic feedback is not obvious (Table 1). But the general feeling when flying with feedbacks is that it is clearly easier to estimate the proximity of the obstacles. 


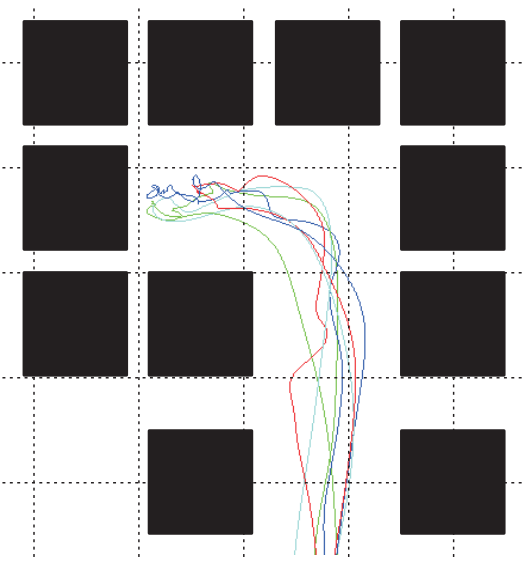

(a)

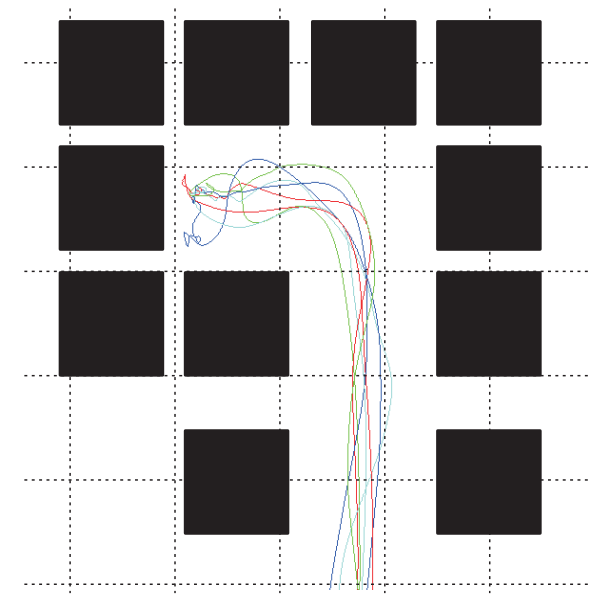

(b)

Figure 16 Confined zone - approach on constant glide: (a) without force feedback; and $(b)$ with VFF feedback

Table 1 Results for confined zone

\begin{tabular}{lcc}
\hline Feedback & $\begin{array}{c}\text { Minimal distance } \\
\text { from end block } \\
\text { (hover point), } \mathrm{m}\end{array}$ & $\begin{array}{c}\text { Average minimal distance } \\
\text { from all obstacles, } \mathrm{m}\end{array}$ \\
\hline Absent & 8.47 & 29.46 \\
With VFF & 8.26 & 29.52 \\
\hline
\end{tabular}

The overall workload is not lowered because the pilot has to apply corrections on the stick that he/she probably would not have done without feedback. Once again, a real benefit is to have the information of the proximity of an obstacle located backwards of the helicopter or outside the field of view. In order to increase the difficulty of this task and, maybe, to have an improved insight of the advantages of haptic functions in this flight condition, the confined area should be downsized.

As it was relatively easy to perform this scenario without feedback, a second piloting task has been evaluated by adding a thick fog in the visual environment. In these conditions, the obstacles could be seen only at a very short distance as shown in Fig. $17 a$. As approaching on a constant glide slope was too difficult in these conditions, the initial speed was a 10-knot level flight. Example trajectories with and without haptic feedback are shown in Fig. $17 b$. 


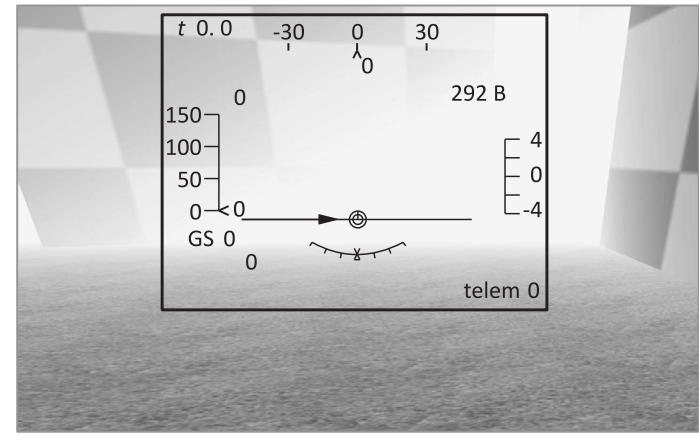

(a)

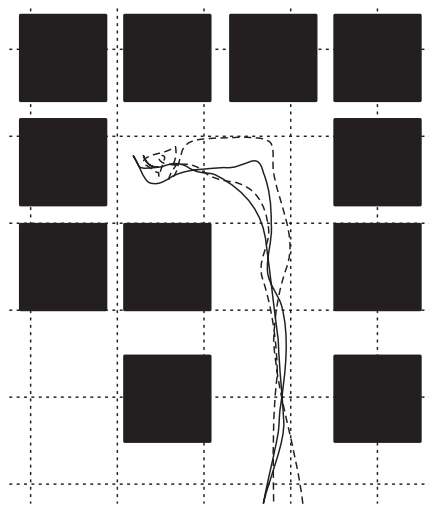

(b)

Figure 17 Confined zone in DVE (fog): (a) in-flight view; and (b) trajectories without feedback (dashed curve) and with VFF feedback (solid curve)

In these conditions, the average minimal distance with respect to all obstacles is lower without feedback ( $29.27 \mathrm{~m}$ vs. $32.27 \mathrm{~m}$ with feedback): as a consequence, there is an increased risk of collision with an obstacle that the pilot cannot see (in the back of the helicopter, for example) when no force feedback is provided.

\section{CONCLUDING REMARKS}

A specific terrain database has been developed, integrating obstacle field navigation benchmarks. As a result of previous works, the tactile cueing used on the stick was a force bias. But two logics have been developed to generate it. These two logics were implemented and evaluated on four different tasks on the PycsHel prototyping and simulation environment at ONERA: the first one is based on force gradients surrounding obstacles, whereas the other one is partially based on $\tau$-theory.

A total of 179 runs have been performed over two months in order to evaluate the benefits of using haptic feedback for obstacle avoidance. All simulation flights were done by one flight dynamics engineer. Further trials will involve actual helicopter pilots in order to get their feedback and expertise, in the framework of the ONERA/DLR cooperation.

It can be concluded that depending on the task performed, the force feedback logics used should be different. For emergency and/or high-speed avoidance 
manoeuvers, the GA gives good results, providing tactile cues at a well suited distance from the obstacle (mainly, on the lateral cyclic control input).

For multiple/close obstacles and low helicopter speeds, the VFF approach is more suitable. Since the force bias is sent on both lateral and longitudinal cyclic control axes, it helps the pilot to "feel" the proximity of obstacles located behind the helicopter or outside his/her field of view. Up to now, this approach is not adapted to high speeds. Combining these two approaches in a single haptic function could cover a large range of situations.

\section{REFERENCES}

1. Watanabe, Y., P. Chavent, C. Seguin, H. de Plinval, T. Loquen, M. Sanfourche, H. Piet Lahanier, L. Planckaert, L. Binet, T. Rakotomamonjy, and B. Le Saux. 2013. PR AZUR Annual Report. ONERA. Technical Report RT 3/18891 DCSD.

2. Demaret, B., and K. Pahlke. 2014. Rotorcraft Research Annual Report. ONERADLR Technical Report.

3. Mettler, B., Z. Kong, C. Goerzen, and M. Whalley. 2010. Benchmarking of obstacle field navigation algorithms for autonomous helicopters. 66th Annual Forum of the American Helicopter Society. Phoenix, AZ.

4. Lee, D. 1998. Guiding movement by coupling taus. Ecol. Psychol. 10(3-4):221-250.

5. Padfield, G., G. Clark, and A. Taghizad. 2005. How long do pilots look forward? Prospective visual guidance in terrain-hugging flight. 31st European Rotorcraft Forum. Florence, Italy.

6. Benoit, B., A.-M. Dequin, K. Kampa, W. Grunhagen, P.-M. Basset, and B. Gimonet. 2000. HOST, a general helicopter simulation tool for Germany and France. 56th Annual Forum of the American Helicopter Society.

7. Von Grünhagen, W., M. Müllhäuser, M. Abildgaard, and R. Lantzsch. 2010. Active inceptors in FHS for pilot assistance systems. 36th European Rotorcraft Forum. Paris, France.

8. Abildgaard, M., L. Binet, A. Taghizad, and W. Von Grünhagen. 2009. VRS avoidance as active function on side-sticks. 65th Annual Forum of the American Helicopter Society. Grapevine, TX.

9. Khatib, O. 1986. Real-time obstacle avoidance for manipulators and mobile robots. Int. J. Robot. Res. 5(1):90-98.

10. Borenstein, J., and Y. Koren. 1989. Real-time obstacle avoidance for fast mobile robots. Trans. Syst. Man Cybernetics 19(5):1179-1187.

11. Carloni, R., V. Lippiello, M. D'Auria, M. Fumagalli, A. Y. Mersha, S. Stramigioli, and B. Siciliano. 2013. Robot vision: Obstacle-avoidance techniques for unmanned aerial vehicles. IEEE Robot. Autom. Mag. 20(4):22-31. 
12. Dozier, G., A. Homaifar, S. Bryson, and M. Bikdash. 1998. Artificial potential fieldbased motion planning/navigation, dynamic constrained optimization and simple genetic hill climbing. Simulation 71(3):168-181.

13. Sarkar, S., S. Reynolds, and E. Hall. 2007. Virtual force field based obstacle avoidance and agent based intelligent mobile robot. Intelligent robots and computer vision XXV: Algorithms, techniques, and active vision. Proc. SPIE. Eds. D. P. Casasent, E. L. Hall, and J. Röning. Vol. 6764. 12 p. 